\title{
Modelo para estimativa da força e torque muscular durante a abdução do ombro
}

\author{
Daniel Cury Ribeiro \\ Marcelo Gregis Estivalet \\ Jefferson Fagundes Loss
}

Universidade Federal do Rio Grande do Sul

Porto Alegre

Brasil

https://doi.org/10.5628/rpcd.08.03.321

\section{RESUMO}

Identificar a força produzida pelos músculos do ombro é essencial para melhor compreender os mecanismos de lesão desta articulação. O objetivo deste estudo foi aplicar um modelo matemático para estimar a força e torque de cada músculo durante o movimento de abdução máxima no plano coronal. Um indivíduo do sexo masculino, 28 anos de idade, 1,78 metros de altura e $80 \mathrm{~kg}$ participou neste estudo. O modelo de otimização foi estruturado no software Matlab $7.0 \AA$

(Mathworks, Inc.) e considerou os seguintes músculos abdutores: deltóide anterior, deltóide médio, deltóide posterior, supraespinal, infraespinal e subscapular. A comparação entre torque e força muscular estimado foi feita através do teste de Kruskal-Wallis. O teste post-hoc de Friedman foi utilizado para identificar diferenças significativas $(\alpha=0,05)$. O músculo deltóide médio apresentou o maior pico de torque estimado $(16,7$ $\mathrm{Nm})$, quando comparado com os outros músculos $(\mathrm{p}<0,05)$. O pico de torque e a força muscular estimados para o supraespinal $(6,6 \mathrm{Nm}$ e $371 \mathrm{~N}$, respectivamente) foram menores que aqueles associados às três porções do deltóide (anterior, médio e posterior). Os resultados do modelo são coerentes com os dados encontrados na literatura e provêem informações importantes acerca da força muscular produzida durante a abdução do ombro.

Palavras-chave: músculo, ombro, reabilitação

\section{ABSTRACT \\ Model for muscle force and moment prediction during the shoulder abduction}

To identify the muscle force produced by the shoulder muscles is essential to improve the knowledge of injuries mechanisms of the shoulder joint. The aim of the present study was to apply a model to estimate the muscle force and torque, by means of a biomechanical model, during shoulder maximal abduction on the coronal plane. One male, 28 years, 1.78 meters and $85 \mathrm{~kg}$ participated in the present study. The optimization model was structured with software Matlab $7.0 \AA$ (MathWorks, Inc.). The model considered as abductor muscles the following: anterior deltoideus, medium deltoideus, posterior deltoideus, supraspinatus, infraspinatus and subscapularis muscles. The comparison between muscle estimated force and moment was performed by the Kruskal-Wallis test, together with the Friedman post hoc test $(\alpha=0.05)$. The medium deltoideus presented the largest estimated torque $(16.7 \mathrm{Nm})$, when compared with the other muscles. The peak torque and force of supraspinatus $(6,6 \mathrm{Nm}$ e $371 \mathrm{~N}$, respectively) was smaller when compared to the three portions of the deltoideus muscle. The results of the model are coherent with those found in the literature and present important information about muscle force production during shoulder abduction.

Key-words: muscle, shoulder, rehabilitation 


\section{INTRODUÇÃOO}

Os músculos do manguito rotador são responsáveis pela estabilização dinâmica da articulação glenoumeral(15), reduzindo consideravelmente os níveis de translação do úmero em relação à glenóide( ${ }^{(9)}$.

Durante a abdução do braço, os músculos supraespinal e deltóide formam um conjunto de forças, em que o primeiro provê forças compressivas na articulação gleno-umeral contrariando as forças de cisalhamento produzidas pelo deltóide(13, 32). Além disso, os músculos do manguito rotador são frequentemente envolvidos em disfunções da articulação do ombro(26).

Ao longo da reabilitação de diferentes disfunções desta articulação, exercícios de reforço muscular para o manguito rotador são comumente recomendados $(10,32,36,37)$. Os programas de reabilitação de atletas envolvem ainda reforço dos músculos do manguito rotador com o ombro abduzido em $90^{\circ}$, devido à grande sobrecarga que esta articulação sofre nesta posição em esportes de arremesso(16,36). Identificar a força produzida pelos músculos do ombro durante gestos desta articulação é essencial para melhor compreender os seus mecanismos de lesão(11). Identificar padrões de recrutamento motor do manguito rotador, bem como, padrões do ritmo escápulo-umeral vem sendo o objectivo de diferentes pesquisadores $(6,14,22,24,25,28)$. Alguns estudos dedicaram-se a identificar o grau de envolvimento de cada músculo no gesto analisado durante rotações internas e externas do ombro $(5,7,11,29)$. Outros voltaram-se para a análise das forças musculares resultantes durante os movimentos referidos $(17,30,31)$. Muitos estudos mediram a magnitude do braço de alavanca de abdução dos músculos do ombro(12, 18, 21, 27). No entanto, os resultados destes estudos divergem, principalmente, quanto à função do supraespinal e do deltóide posterior ao longo da abdução.

Determinados estudos apontam o supraespinal como um importante abdutor ao longo de toda a amplitude de movimento, ao contrário de outros que sugerem que este músculo tem maior função abdutora apenas no início do movimento(35). Além disso, os resultados do estudo de Hughes e An(11) sugerem que gestos de abdução do ombro parecem não provocar grandes sobrecargas na estrutura músculo-ten- dinosa do supraespinal. O presente estudo teve como objetivo aplicar um modelo matemático para estimar a força e o torque de cada músculo durante o movimento de abdução máxima no plano coronal.

\section{MÉTODOS}

\section{Amostra}

A amostra foi constituída por um sujeito, do sexo masculino, 28 anos de idade, 1,78 metros de altura e $85 \mathrm{~kg}$. Antes de qualquer envolvimento com este trabalho, o indivíduo assinou o Termo de Consentimento Informado, consentindo formalmente na participação desta pesquisa.

\section{Modelo de Otimização}

O modelo de optimização foi estruturado no software Matlab 7.0 ® (Mathworks, Inc.). Os músculos considerados como abdutores foram: deltóide anterior (DA), deltóide médio (DM), deltóide posterior (DP), supraespinal (SE), infraespinal (IE) e subscapular (SUBS), conforme estudo de Kuechle et al. (18). As variáveis independentes do modelo são: torque (medido pelo dinamómetro isocinético), ângulo de abdução (medido através de um eletrogoniómetro), braço de alavanca de cada músculo abdutor, área de secção transversa fisiológica (ASTF) e valor de tensão específica (s) dos músculos do ombro; enquanto que as variáveis dependentes do modelo são a força e o torque estimados dos músculos abdutores do ombro. Os dados de braço de alavanca(18), $\operatorname{ASTF}^{(33)}$ e $\mathrm{s}^{(7)}$ foram obtidos da literatura. Os valores de braço de alavanca dependem do ângulo de abdução do ombro e, desta maneira, a cada ângulo de abdução corresponde um valor do braço de alavanca pode adoptar um novo valor(18). A tensão específica é uma constante de proporcionalidade entre a área de secção transversa fisiológica e a força máxima produzida pelo músculo(5). O valor de $s$ adoptado foi de 70 $\mathrm{N} / \mathrm{cm}^{2}$ (7). De forma geral, o modelo pretende encontrar o nível de força que cada músculo necessita produzir para o conjunto do torque dos músculos equivaler ao torque medido no dinamómetro isocinético. Para isso, o modelo opera com as variáveis, conforme descrito a seguir.

$O$ produto entre ASTF e s permite uma estimativa da força máxima que o músculo pode produzir [Equação 1], é a denominada força potencial $(F p)^{(21)}$. 
$F p_{i}=A S T F_{i} \times \sigma$

\section{Em que:}

$i=1,2,3, \ldots$ n: índice do músculo abdutor;

$F p_{i}=$ força potencial do músculo "i";

$\mathrm{ASTF}_{i}=$ área de secção transversa fisiológica do músculo "i";

$\sigma=$ tensão específica;

O músculo com maior ASTF é considerado como o músculo referência $(\mathrm{u})$, consequentemente:

$F u=A S T F_{u} \times \sigma$

Da mesma forma que a força potencial, o torque potencial $(T p)$ pode ser estimado como sendo o produto do braço de alavanca e da $F p$ [Equação 3] (4).

$T p_{i}=b r a c ̧ o_{i} \times F p_{i}$

\section{Em que:}

$i=1,2,3, \ldots$ n: índice do músculo abdutor;

$T p_{i}=$ torque potencial do músculo "i";

$F p_{i}=$ força potencial do músculo "i";

braço $_{i}=$ braço de alavanca do músculo "i";

A partir do $T p$, o modelo classifica os músculos motores primários e secundários. Músculos com $T p$ maior que a magnitude do produto da média de $T p$ de todos músculos e um factor limiar são considerados primários. Músculos com menor Tp que este produto são considerados como motores secundários. O factor limiar tem uma função fundamental no algoritmo do modelo, na medida em que ele determina quais os músculos que serão motores primários e quais os que serão motores secundários. $\mathrm{O}$ valor de 0.9 foi adotado para o factor limiar, o qual foi adoptado por dados obtidos na literatura.

Foi assumido que o torque abdutor medido no dinamómetro isocinético é igual à soma dos torques de todos os músculos abdutores do ombro [Equação 4] (20).

$T m=\sum_{i=1}^{n} \operatorname{Tmusc}_{i}$

Em que:

$\mathrm{Tm}=$ torque medido;

Tmusc $_{i}=$ torque muscular do músculo "i";
O torque muscular será de fato, uma fracção do $T p$ [equação 3], conforme demonstrado na equação [5].

$\operatorname{Tmusc}_{i}=\Delta \times T p_{i}$

Em que:

Tmusc $_{i}=$ torque muscular do músculo "i";

$\mathrm{D}=0 ; 0,05 ; 0,1 ; 0,15 ; \ldots 1$;

$\mathrm{Tp}_{i}=$ torque potencial do músculo "i".

O incremento (D) permite modular o torque muscular produzido pelos músculos abdutores do ombro, com a finalidade de ajustar a força que os músculos abdutores devem produzir.

A ASTF de cada músculo foi normalizada pela maior ASTF (ASTFu) (7). A normalização das ASTF resulta em um quociente $(Q)$ [Equação 5].

$Q_{i}=\frac{A S T F_{i}}{A S T F_{u}}$

A equação (4) pode ser combinada com a equação [5], resultando em:

$T m=\left(\sum_{i=1}^{n} b_{i}{ }^{\perp} \times F p_{i}\right)$

Substituindo $F p$ na Equação 6 pela equação [1] resulta em:

$T m=\Delta \sum_{i=1}^{n} b_{i}^{\perp} \times A S T F_{i} \times \sigma$

Conforme comentado anteriormente, o incremento (D) permite modular o torque muscular produzido pelos músculos abdutores do ombro. $\mathrm{O}$ incremento permite modular a força produzida pelos músculos abdutores do ombro. Ela varia de 0 à 1 , com intervalos de 0,05. Esta modulação ocorre para cada grau da amplitude de movimento de abdução. Normalizando a ASTF, conforme citado anteriormente:

$T m=\Delta \times F u \times\left(\sum_{i=1}^{n} b_{i}{ }^{\perp} \times Q_{i}\right)$

Em que:

$\mathrm{D}=0 ; 0,05 ; 0,1 ; 0,15 ; \ldots 1$;

$F u=$ força do músculo (u) armazenada para cada

ângulo correspondente;

O modelo assume que o torque externo é neutraliza- 
do, principalmente, pelos motores primários. Assim, a equação [8] é aplicada inicialmente apenas aos músculos motores primários [Equação 9].

$\operatorname{Tmp}=\Delta_{p} \times F u \times\left(\sum_{i=1}^{n} b_{i}{ }^{\perp} \times Q_{i}\right)$

Em que:

$\Delta_{P}=$ incremento dos músculos abdutores primários; $i=1,2,3, \ldots \mathrm{m}$ : índice do músculo abdutor motor primário;

$T m p=$ torque global dos músculos motores primários;

A magnitude de força muscular seleccionada pelo modelo é aquela que promove o menor torque remanescente positivo entre o torque medido e o torque calculado [Equação 10].

$\operatorname{Tr}=\operatorname{Tmp}-\operatorname{Tm}$

Em que:

$\operatorname{Tr}=$ torque remanescente;

De seguida, as equações [9] e [10] são aplicadas aos músculos motores secundários, com a restrição de que a soma dos torques dos músculos motores secundários deve igualar o torque remanescente $(\mathrm{Tr})$.

$\operatorname{Tr}=\operatorname{Tms}=\Delta_{S} \times F u \times\left(\sum_{i=1}^{n} b_{i}{ }^{\perp} \times Q_{i}\right)$

$E m=T m s-T r$

Em que:

$\Delta_{S}=$ incremento dos músculos abdutores secundários; $i=1,2,3, \ldots \mathrm{k}$ : índice do músculo abdutor motor secundário;

Tms = torque global dos músculos motores secundários;

$E m$ = erro mínimo;

Assim, com os valores de $F u$ armazenados é possível estimar, através da Equação [13], qual a força que cada músculo produziu para cada valor do ângulo abdução.

$F_{i}=Q_{i} \cdot F u$

$i=1,2, \ldots$ n: índice do músculo abdutor;
$F_{i}=$ força do músculo i;

$Q_{i}=$ quociente do músculo i;

$F u=$ força do músculo (u) armazenada para cada ângulo correspondente;

O valor de D que promova a menor diferença positiva entre o Tms e Tr é seleccionada como a mais adequada e, determinará assim, a magnitude da força dos músculos motores secundários.

\section{Coleta de dados}

A coleta consistiu na mensuração do torque máximo de abdução e adução do ombro a $60^{\circ} / \mathrm{s}$ no plano coronal. Para tal, foi utilizado um dinamómetro isocinético, marca Cybex, modelo Norm (Dataq Instruments, Inc. Ohio - EUA). Com a finalidade de registar com maior precisão a posição articular, foi utilizado um eletrogoniómetro da marca Biomectrics Ltd (Cwmfelinfach, Reino Unido), modelo XM 180, adaptado junto ao dinamômetro isocinético. O dinamómetro isocinético e o eletrogoniómetro estavam conectados a um computador através de um conversor analógico-digital de 16 canais (Computer Boards, CIO-DAS 16).

Previamente ao teste, o indivíduo realizou aquecimento dos músculos do membro superior direito. Além disso, o sujeito realizou três repetições de contrações submáximas para adução e abdução de ombro, como familiarização ao teste, o qual consistia de cinco contracções máximas de abdução e adução de ombro até $90^{\circ}$ de abdução. A velocidade angular foi ajustada em $60^{\circ}$ s Durante o teste, o indivíduo permaneceu sentado no aparelho isocinético, conforme sugestão do fabricante.

\section{Processamento dos dados}

Para tratamento dos dados foram utilizados o software SAD32 (Sistema de Aquisição de Dados, desenvolvido pelo Laboratório de Medições Mecânicas da Universidade Federal do Rio Grande do Sul) e rotinas desenvolvidas em ambiente Matlab 7.0 ${ }^{\circledR}$ (MathWorks Inc, Massachusetts - EUA).

\section{Análise Estatística}

Foi feita a comparação entre os torques estimados de cada músculo e uma comparação entre a força estimada de cada músculo. Esta comparação entre os 
valores de torque estimado dos músculos e a comparação de força estimada dos músculos foi feita através do teste de Kruskal-Wallis. O teste post-hoc de Friedman foi utilizado para identificar diferenças significativas, considerando $\alpha=0,05$. Para isso, foi utilizado o programa Matlab 7.0 ${ }^{\circledR}$ (MathWorks Inc, Massachusetts - EUA).

\section{RESULTADOS}

Na mesma Tabela 1, encontram-se os dados de braço de alavanca e área de secção transversa fisiológica para cada músculo abdutor analisado.

Tabela 1. ASTF a , braço de alavanca ${ }^{\mathrm{b}}$ de cada músculo abdutor.

\begin{tabular}{lcccccc}
\hline & DA & DM & DP & Supra & Infra & Sub \\
\hline ASFT $\left(\mathrm{cm}^{2}\right)$ & 7,38 & 9,08 & 9,45 & 5,21 & 9,51 & 13,51 \\
\hline Braço da & 0,237 & 0,0294 & 0,0300 & 0,0345 & 0,0122 & 0,0089 \\
Alavanca (m) & & & & & & \\
\hline
\end{tabular}

\begin{tabular}{lllllll}
\hline Pico & $\left(91^{\circ}\right)$ & $\left(91^{\circ}\right)$ & $\left(91^{\circ}\right)$ & $\left(91^{\circ}\right)$ & $\left(12^{\circ}\right)$ & $\left(78^{\circ}\right)$ \\
\hline
\end{tabular}

aASTF: área de secção transversa fisiológica - extraído de (33) bBraço de alavanca - extraído de (19).

DA: deltóide anterior; DM: deltóide médio; DP: deltóide posterior; Supra: supraespinal; Infra: infraespinal; Subs: subscapular.

O valor do pico de Tm está apresentado na Tabela 2, bem como, os valores de torque e força estimados de cada músculo.

Tabela 2. Torque e força de cada músculo analisado.

\begin{tabular}{lcccccc}
\hline & DA & DM & DP & Supra & Infra & Sub \\
\hline Torque $(\mathrm{Nm})$ & 10,3 & 16,7 & 12,4 & 6,6 & 0,07 & 0,14 \\
Pico & $\left(64^{\circ}\right)$ & $\left(56^{\circ}\right)$ & $\left(80^{\circ}\right)$ & $\left(33^{\circ}\right)$ & $\left(29^{\circ}\right)$ & $\left(68^{\circ}\right)$ \\
\hline Força $(\mathrm{N})$ & 525 & 647 & 673 & 371 & 14 & 19 \\
Pico & $\left(43^{\circ}\right)$ & $\left(43^{\circ}\right)$ & $\left(43^{\circ}\right)$ & $\left(43^{\circ}\right)$ & $\left(43^{\circ}\right)$ & $\left(43^{\circ}\right)$ \\
\hline
\end{tabular}

DA: deltóide anterior; DM: deltóide médio; DP: deltóide posterior; Supra: supraespinal; Infra: infraespinal; Subs: subscapular.

O comportamento e magnitude do torque medido encontra-se na Figura 1. O pico de $\mathrm{Tm}$ foi de $41 \mathrm{Nm}$. O ângulo correspondente ao pico de $\mathrm{Tm}$ foi $62^{\circ}$ de abdução (Figura 1).

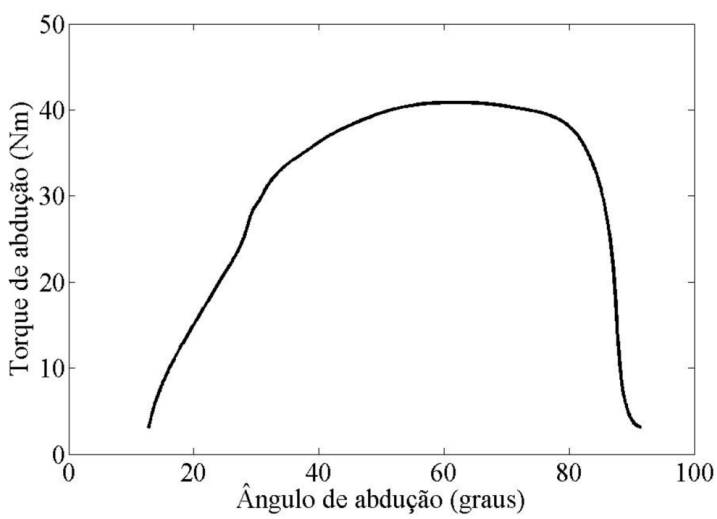

Figura 1. Torque medido de abdução em função do ângulo de abdução do ombro.

Os músculos considerados como motores primários da abdução foram DA, DM, DP, Supraespinal; sendo os músculos Infraespinal e Subscapular os motores secundários dentre os músculos analisados. $\mathrm{O}$ erro mínimo médio foi de $0,17 \pm 0,17 \mathrm{Nm}$ (Figura 2).

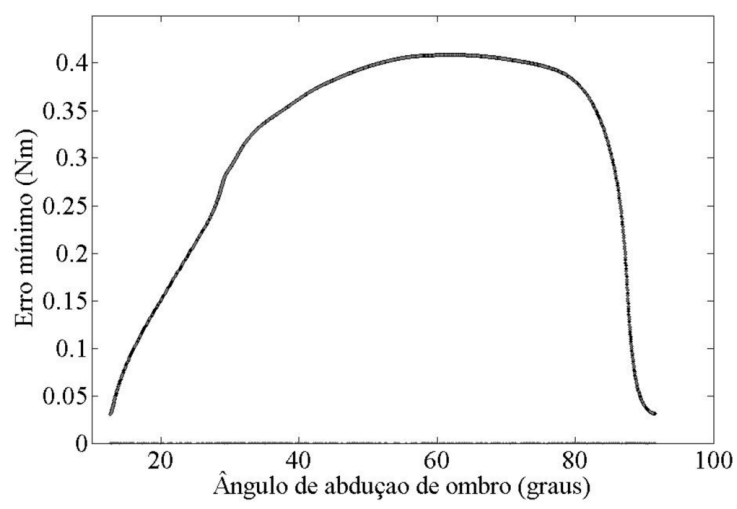

Figura 2. Erro mínimo em função do ângulo de abdução do ombro.

O comportamento do torque estimado dos músculos variou conforme o músculo analisado (3). Foram identificadas diferenças significativas entre os torques estimados de todos músculos abdutores do ombro, com excepção dos músculos DA e DP para um nível de significância de $\mathrm{p}<0,05$. O pico de torque muscular estimado foi de $16,7 \mathrm{Nm}$ para o músculo DM. Os músculos DA e DP apresentaram torques estimados muito similares: picos de 10,3 e 12,4 
Nm, respectivamente, não havendo diferenças estatisticamente significativas entre os valores de pico dos torques estimados destes músculos. O músculo Supraespinal apresentou magnitudes abaixo das porções do deltóide. O músculo Infraespinal apresentou o menor pico de torque $(0,07 \mathrm{Nm})$.

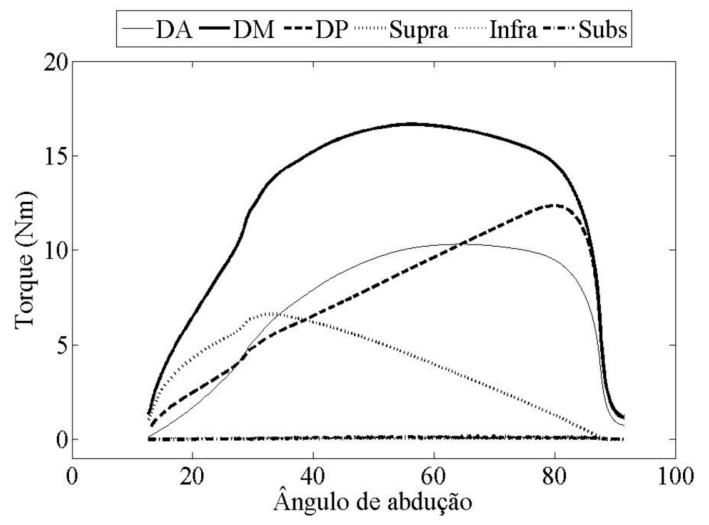

DA: deltóide anterior; DM: deltóide médio; DP: deltóide posterior; Supra: supraespinal; Subs: subscapular; Infra: infraespinal.

Figura 3. Torque dos músculos abdutores.

Os músculos DA, DM, DP e Supraespinal apresentam comportamento de força muito similar, divergindo apenas na magnitude da força produzida (4). A comparação entre os valores de força muscular estimada, ao longo da amplitude de movimento $\mathrm{O}_{2}$ evidenciou diferenças significativas para a força muscular estimada entre todos os músculos $(p<0,05)$, com excepção dos músculos DM e DP, os quais apresentaram níveis de força muito similares (4 e Tabela 2). Os músculos DM e DP apresentam as maiores magnitudes de força durante a abdução, com pico de força $673 \mathrm{~N}$ e $647 \mathrm{~N}$, respectivamente. Além disso, todos os músculos atingiram o pico de força para o mesmo valor do ângulo de abdução (Tabela 2). A força estimada para o músculo Infraespinal foi muito baixa, e o mesmo atingiu um pico de força de apenas $14 \mathrm{~N}$. O Supraespinal apresentou um pico de força de $371 \mathrm{~N}$ e manteve níveis similares de força ao longo de boa parte da amplitude de movimento.

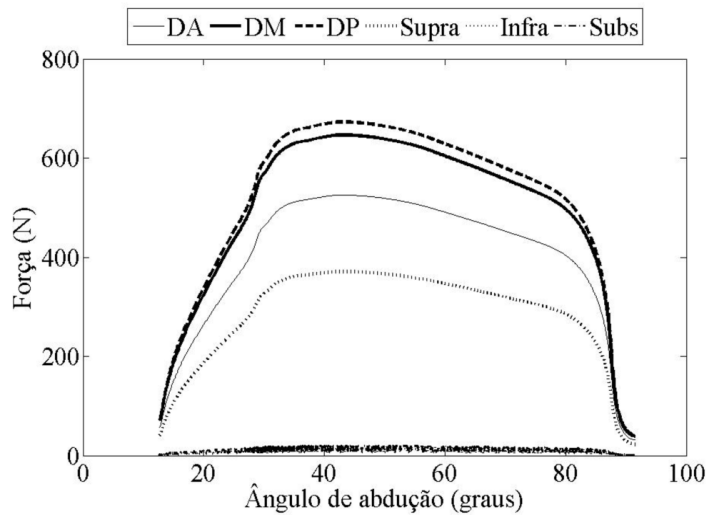

DA: deltóide anterior; DM: deltóide médio; DP: deltóide posterior; Supra: supraespinal; Subs: subscapular; Infra: infraespinal.

Figura 4. Força dos músculos abdutores.

\section{DISCUSSÃO}

Torque é a medida da tendência de que uma força tem em gerar movimento angular(38). Pela simples definição de torque, é possível estabelecer que os músculos com maior capacidade de produção de torque serão classificados como motores primários ou secundários(27). Isso justifica a classificação de todas as porções do deltóide e o músculo Supraespinal como motores primários. $\mathrm{O}$ torque potencial reflectirá a tendência do comportamento do braço de alavan$\mathrm{ca}^{(19)}$. Com base no comportamento do torque e força muscular do Supraespinal, parece que este músculo tem maior capacidade de abdução durante o início do movimento e, posteriormente, torna-se um estabilizador da articulação gleno-umeral. Os nossos resultados estão de acordo com aqueles de outros autores ${ }^{(18,}$ 27) que afirmam que o músculo Supraespinal é um importante abdutor no início do movimento e que, em seguida, comporta-se como estabilizador da cabeça do úmero contra a glenóide. A manutenção da magnitude de força deste músculo, ao longo do movimento, vai de encontro a estas afirmações. No entanto, outros autores $(8,12)$ afirmam que o músculo Supraespinal é um importante abdutor ao longo de toda a amplitude de movimento. Estes diferentes resultados são consequências das diferenças metodológicas utilizadas em cada estudo(18). 
Apesar de não contribuir para o torque muscular de abdução como os deltóides, a produção de força do Supraespinal ao longo da abdução foi de, em média, 280 N. A força muscular produzida gera tensão na estrutura tendínea(11), assim, independentemente da sua função como músculo motor primário ou estabilizador durante a abdução de ombro, o tendão do músculo Supraespinal está exposto à sobrecarga ao longo de toda a amplitude de movimento avaliada. Isto sugere, claramente, repercussões no plano de tratamento das disfunções músculo-tendíneas do músculo Supraespinal, visto que não parece haver regiões de menor sobrecarga sobre a estrutura músculo-tendínea (a não ser os extremos de amplitude). O maior envolvimento do músculo Supraespinal, se comparado ao músculo Infraespinal, durante a elevação do braço foi também observada em estudos eletromiográficos (3). A função dos músculos Infraespinal e Subscapular durante a abdução não é muito clara na literatura (21). De acordo com Otis et al. (27), músculos com braço de alavanca menor que um centímetro podem ser considerados estabilizadores do movimento. Assim, os músculos Infraespinal e Subscapular são considerados como estabilizadores da articulação gleno-umeral durante este movimento ${ }^{(13,21)}$. Apesar do algoritmo do modelo não dar ênfase à função muscular de estabilização articular da articulação gleno-umeral, o baixo torque produzido pelos músculos Subscapular e Infraespinal permite afirmar que estes músculos, realmente, apresentam uma função de estabilizadores da articulação gleno-umeral.

Comparações directas das magnitudes de força e torque muscular com outros estudos são prejudicadas em virtude das diferenças na estrutura metodológica do estudo, bem como, da estrutura do modelo utilizado. No entanto, algumas observações podem ser feitas. Os resultados de força apresentados neste estudo são similares ao encontrado por outros autores(11), os quais estimaram a força isométrica de abdução dos músculos do ombro a partir do torque isométrico de abdução (medido num dinamómetro isocinético). A força isométrica máxima dos músculos DA (323 N), DM (434 N) e Supraespinal (117 $\mathrm{N})$ são similares aos valores médios encontrados no nosso estudo. Ao contrário, os resultados de força para os músculos Infraespinal (205 N), Subscapular
$(283 \mathrm{~N})$ e DP $(0 \mathrm{~N})$ divergem dos nossos resultados. Certamente, a diferença da estrutura do modelo de optimização tem papel fundamental nestas diferenças. Além disso, estes autores encontraram uma função adutora para o músculo DP. A função adutora ou abdutora do DP justifica-se pela simplificação das subdivisões do músculo deltóide. Estudos eletromiográficos apresentam resultados divergentes, sugerindo que o DP é abdutor (23), enquanto outro estudo sugere justamente o contrário ${ }^{(34)}$. O nosso estudo considerou o músculo deltóide como composto por três porções (anterior, médio e posterior) e respectivos braços de alavanca, e os outros músculos como possuindo apenas um braço de alavanca; no entanto, sabe-se que os músculos podem ser divididos em muitas subunidades ${ }^{(34)}$. Entretanto, estas simplificações são necessárias para tornar possível a estimativa da força muscular(2). Evidentemente, isso interfere nos resultados do trabalho, porém, a subdivisão de músculos em outras subunidades funcionais exigiria um maior número de braços de alavanca para cada porção muscular; isto aumentaria ainda mais a complexidade da estrutura do modelo.

O presente estudo aplicou um modelo de optimização para a abdução do ombro. Este estudo apresenta algumas limitações: (1) apenas os músculos DA, DM, DP, Supraespinal, Infraespinal e Subscapular foram incluídos no modelo; (2) os dados de torque de entrada do modelo são provenientes de um único indivíduo; (3) este estudo não utilizou restriçõos de força articular para a estimativa da força muscular. Devido à simplificação adotada pelo modelo, os músculos motores primários apresentam um comportamento de força similar entre si. Isto deve-se especificamente à Equação [9] do algoritmo utilizado no modelo [7]. O item (3) tem uma importância muito grande na medida em que as forças translacionais articulares excessivas provocariam subluxações ou luxações articulares. O acréscimo de novas restrições tende a aumentar a estimativa da força muscular(11), mas por outro lado, tende a aumentar a incerteza da estimativa da força e torque musculares ${ }^{(1)}$. Podemos afirmar que o modelo apresenta resultados coerentes com os dados encontrados na literatura e, apesar de ser baseado em valores de um único indivíduo, este trabalho provê importantes informações referentes à produção de força dos músculos do 
ombro durante o movimento de abdução desta articulação, em especial, em relação aos músculos do manguito rotador. A aplicação do modelo a informações de torque provenientes de uma amostra mais ampla permitirá a obtenção de resultados ainda mais conclusivos.

\section{Agradecimentos}

Este estudo recebeu apoio financeiro da Coordenação de Aperfeiçoamento de Pessoal de Nível Superior (CAPES) - Brasil.

\section{CORRESPONDÊNCIA}

\section{Daniel Cury Ribeiro}

R: Barão do Amazonas 793/ 401

Bairro Jardim Botânico

CEP: 90670-003

Porto Alegre - RS - Brasil

Telefone: 51-33301546/91438270

E-mail: daniel.cury.ribeiro@gmail.com

\section{REFERÊNCIAS}

1. Allard P, Blanchi J-P, Aïssaoui R (1995). Bases of ThreeDimensional Reconstruction. In: Three-Dimensional Analysis of Human Motion, P. Allard, I. Stokes, and J-P Blanchi, eds., Human Kinetics, Champaign, IL, 19-40.

2. An KN, Kaufman KR, Chao EY-S (1995). Estimation of Muscle and Joint Forces. In: Three-dimensional analysis of human movement, Human Kinetics, Champaign, IL.

3. Ballantyne BT, O'Hare SJ, Paschall JL, Pavia-Smith MM, Pitz AM, Gillon JF, Soderberg GL (1993).

Electromyographic activity of selected shoulder muscles in commonly used therapeutic exercises. Phys Ther 73(10): 668-677; discussion 677-682.

4. Bassett RW, Browne AO, Morrey BF, An KN (1990). Glenohumeral muscle force and moment mechanics in a position of shoulder instability. J Biomech 23(5): 405-415.

5. Chang YW, Hughes RE, Su FC, Itoi E, An KN (2000). Prediction of muscle force involved in shoulder internal rotation. J Shoulder Elbow Surg 9(3): 188-195.

6. Ebaugh DD, McClure PW, Karduna AR (2005). Threedimensional scapulothoracic motion during active and passive arm elevation. Clin Biomech (Bristol, Avon) 20(7): 700709.

7. Favre P, Sheikh R, Fucentese SF, Jacob HA (2005). An algorithm for estimation of shoulder muscle forces for clinical use. Clin Biomech (Bristol, Avon) 20(8): 822-833.

8. Graichen H, Englmeier KH, Reiser M, Eckstein F (2001). An in vivo technique for determining 3D muscular moment arms in different joint positions and during muscular activation - application to the supraspinatus. Clin Biomech (Bristol, Avon) 16(5): 389-394.

9. Graichen H, Stammberger T, Bonel H, Karl-Hans E, Reiser M, Eckstein F (2000). Glenohumeral translation during active and passive elevation of the shoulder - a 3D openMRI study. J Biomech 33(5): 609-613.

10. Hayes K, Callanan M, Walton J, Paxinos A, Murrell GA (2002). Shoulder instability: management and rehabilitation. J Orthop Sports Phys Ther 32(10): 497-509.

11. Hughes RE, An KN (1996). Force analysis of rotator cuff muscles. Clin Orthop Relat Res (330): 75-83.

12. Hughes RE, Niebur G, Liu J, An KN (1998). Comparison of two methods for computing abduction moment arms of the rotator cuff. J Biomech, 31(2): 157-160.

13. Inman VT, Saunders JB, Abbott LC (1944). Observations of the function of the shoulder joint. Journal of Bone and Joint Surgery 26-A: 1-30.

14. Karduna AR, McClure PW, Michener LA, Sennett B (2001). Dynamic measurements of three-dimensional scapular kinematics: a validation study. J Biomech Eng 123(2): 184-190.

15. Kelkar R, Wang VM, Flatow EL, Newton PM, Ateshian GA, Bigliani LU, Pawluk RJ, Mow VC (2001). Glenohumeral mechanics: a study of articular geometry, contact, and kinematics. J Shoulder Elbow Surg 10(1): 7384.

16. Kibler WB, McMullen J, Uhl T (2001). Shoulder Rehabilitation Strategies, Guidelines, and Practice. Orthop Clin North Am 32(3): 527-538.

17. Krug RC, Toledo JM, Castro MP, Ribeiro DC, Martinez FG, Loss JF (2005). Influência de fatores mecânicos e fisiológicos no torque de rotação interna e externa do ombro. XI Congresso Brasileiro de Biomecânica, João Pessoa. 
18. Kuechle DK, Newman SR, Itoi E, Morrey BF, An KN (1997). Shoulder muscle moment arms during horizontal flexion and elevation. J Shoulder Elbow Surg 6(5): 429-439.

19. Kuechle DK, Newman SR, Itoi E, Niebur GL, Morrey BF, An KN (2000). The relevance of the moment arm of shoulder muscles with respect to axial rotation of the glenohumeral joint in four positions. Clin Biomech (Bristol, Avon) 15(5): 322-329.

20. Langenderfer JE, Carpenter JE, Johnson ME, An KN, Hughes RE (2006). A probabilistic model of glenohumeral external rotation strength for healthy normals and rotator cuff tear cases. Ann Biomed Eng 34(3): 465-476.

21. Liu J, Hughes RE, Smutz WP, Niebur G, Nan-An K (1997). Roles of deltoid and rotator cuff muscles in shoulder elevation." Clin Biomech (Bristol, Avon) 12(1): 32-38.

22. Ludewig PM, Cook TM (2000). Alterations in shoulder kinematics and associated muscle activity in people with symptoms of shoulder impingement. Phys Ther 80(3): 276291.

23. McCann PD, Wootten ME, Kadaba MP, Bigliani LU.(1993). A kinematic and electromyographic study of shoulder rehabilitation exercises." Clin Orthop Relat Res (288): 179188.

24. McClure PW, Bialker J, Neff N, Williams G, Karduna A (2004). Shoulder function and 3-dimensional kinematics in people with shoulder impingement syndrome before and after a 6-week exercise program. Phys Ther 84(9): 832848 .

25. McQuade KJ, Dawson J, Smidt GL (1998). Scapulothoracic muscle fatigue associated with alterations in scapulohumeral rhythm kinematics during maximum resistive shoulder elevation. J Orthop Sports Phys Ther 28(2): 74-80.

26. Michener LA, McClure PW, Karduna AR (2003). Anatomical and biomechanical mechanisms of subacromial impingement syndrome. Clin Biomech (Bristol, Avon) 18(5): 369-379.

27. Otis JC, Jiang CC, Wickiewicz TL, Peterson MG, Warren RF, Santner TJ (1994). Changes in the moment arms of the rotator cuff and deltoid muscles with abduction and rotation. J Bone Joint Surg Am 76(5): 667-676.

28. Pascoal AG (2001). Ombro e Elevação do Braço. Análise cinemática e eletromiográfica sobre a influência da carga externa e velocidade do braço no ritmo escápulo-umeral, Tese de Doutorado. Universidade Técnica de Lisboa, Lisboa.

29. Ribeiro DC, Toledo JM, Krug RC, Loss JF (2005). Modelo para estimativa da força dos músculos rotadores internos do ombro. XI Congresso Brasileiro de Biomecânica, João Pessoa.

30. Toledo JM, Krug RC, Castro MP, Ribeiro DC, Loss JF (2006). Differences in the torque and force production during the shoulder external rotation in the transverse and sagittal planes. $V$ World Congress of Biomechanics, Munique Germany.

31. Toledo JM, Ribeiro DC, Loss JF (2006). Critérios mecânicos para progressão de exercícios de rotação interna e externa do ombro no plano sagital. Revista Brasileira de Fisioterapia, (submetido).

32. Tytherleigh-Strong G, Hirahara A, Miniaci A (2001). Rotator cuff disease. Curr Opin Rheumatol 13(2): 135-145.

33. Veeger HE, Van der Helm FC, Van der Woude LH, Pronk GM, Rozendal RH (1991). Inertia and muscle contraction parameters for musculoskeletal modelling of the shoulder mechanism. J Biomech 24(7): 615-629.

34. Wickham JB, Brown JM (1998). Muscles within muscles: the neuromotor control of intra-muscular segments. Eur $J$ Appl Physiol Occup Physiol 78(3): 219-225.

35. Wilk KE, Harrelson GL, Arrigo C, Chmielewski T (2000). Reabilitação do Ombro. In: Reabilitação física das lesões esportivas, JR Andrews, GL Harrelson, and KE Wilk, eds., Guanabara Koogan, Rio de Janeiro, 350-403.

36. Wilk KE, Meister K, Andrews JR (2002). Current concepts in the rehabilitation of the overhead throwing athlete. Am J Sports Med 30(1): 136-151.

37. Wilk KE, Reinold MM, Dugas JR, Andrews JR (2002). Rehabilitation following thermal-assisted capsular shrinkage of the glenohumeral joint: current concepts. J Orthop Sports Phys Ther 32(6): 268-292.

38. Zatsiorsky VM (2002). Kinetics of human motion, Human Kinetics, Champaign, IL. 